\title{
TRATAMENTO CIRÚRGICO DA INSTABILIDADE FÊMORO-PATELAR
}

\author{
SURGICAL TREATMENT OF PATELLOFEMORAL INSTABILITY
}

Marco Antônio Percope de Andrade', Guilherme Moreira de Abreu e Silva², Marcelo Machado Freire², Luiz Eduardo Moreira Teixeira ${ }^{4}$

\section{RESUMO}

Objetivo: Descrever os resultados funcionais do tratamento cirúrgico de pacientes portadores de instabilidade fêmoro-patelar submetidos a realinhamento do aparelho extensor. Métodos: Trata-se de estudo retrospectivo de 34 joelhos operados devido à instabilidade fêmoro-patelar no período de 1989 a 2004. Os pacientes foram avaliados no pós-operatório remoto, sendo aplicado questionário funcional. Resultados: Em um seguimento médio de seis anos e cinco meses, o valor médio de pontuação foi de 82,94 pontos no grupo operado $(p=0,00037)$. Melhora do quadro de dor $(97,05 \%$ de excelentes e bons resultados) e baixa taxa de recorrência dos episódios de luxação $(5,88 \%)$ foram observadas no grupo, embora pontuações mais baixas tenham sido obtidas em atividades de maior demanda articular (agachamento, prática de corridas e execução de saltos). Nenhum dos pacientes avaliados desenvolveu artrose no tempo de seguimento. Conclusão: O procedimento de realinhamento articular descrito neste artigo mostrou-se eficaz no tratamento dos pacientes com instabilidade fêmoro-patelar recidivante.

Descritores - Instabilidade articular; Patela; Joelho

\section{ABSTRACT}

Objective: To describe functional outcomes following surgical treatment of patients with patellofemoral instability submitted to patellar realignment. Methods: This was a retrospective study evaluating 34 operated knees for patellofemoral instability between 1989 and 2004. The patients were evaluated in the late postoperative period when a functional questionnaire was applied. Results: After a mean follow-up time of 6 years and 5 months, the mean score was 82.94 in the surgical group ( $p=0.00037)$. The results of this investigation showed pain relief in $97.05 \%$ and low rate of recurrent dislocation (5.88\%), although lower scores were seen in intense articular activities (squatting, running and jumping). No patient developed osteoarthritis while being followed up. Conclusion: The procedure for joint described in this paper was shown to be effective for treating patients with recurrent patellofemoral instability.

Keywords - Joint instability; Patella; Knee

\section{INTRODUÇÃO}

A instabilidade fêmoro-patelar é ainda um grande desafio para o ortopedista, não havendo consenso sobre a melhor abordagem, se conservadora ou cirúrgica ${ }^{(1,2)}$ e, nos casos de indicação cirúrgica, qual a melhor técnica a ser empregada. Aquelas que envolvem a liberação do retináculo lateral associada à plastia do vasto medial são denominadas realinhamento proximal ${ }^{(3,4)}$ e podem ser realizadas por via aberta ou fechada (artroscópica) ${ }^{(5)}$. As que utilizam procedimentos ósseos sobre a tuberosidade tibial anterior, com o intuito de melhorar a biomecânica articular, são denominadas realinhamento distal ${ }^{(4,6)}$ (Figura 1).

Dejour et $a l^{(7)}$ considerou quatro fatores predisponentes à luxação: displasia troclear, inclinação lateral da patela, interpretada como displasia do vasto medial oblíquo, patela alta e lateralização da tuberosidade tibial anterior (aumento do ângulo "Q"). Nas últimas décadas, tem sido dada maior importância ao ligamento fêmoro-patelar medial como estabilizador articular, sendo seu reparo descrito principalmente nos casos de luxação traumática e na ausência de fatores predisponentes ${ }^{(8,9)}$. Outros procedimentos cirúrgicos a ser considerados, porém em situações de exceção, são as trocleoplastias e as patelectomias parciais ou totais ${ }^{(8)}$.

Didaticamente, podem-se dividir as instabilidades crônicas fêmoro-patelares em quatro grupos: as instabilidades maiores (luxação habitual e inveterada), instabilidades objetivas (luxação recidivante e traumática), instabilidade potencial e artrose fêmoro-patelar ${ }^{(1,6,7)}$.

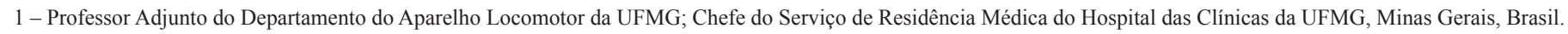

2 - Preceptor de Residência Médica do Serviço de Ortopedia do Hospital das Clínicas da UFMG, Minas Gerais, Brasil.

3 - Ortopedista e Cirurgião de Pé e Tornozelo do Instituto Biocor, Minas Gerais, Brasil.

4 - Preceptor de Residência Médica do Hospital das Clínicas da UFMG; Cirurgião da Oncologia Ortopédica do Hospital das Clínicas da UFMG, Minas Gerais, Brasil. 


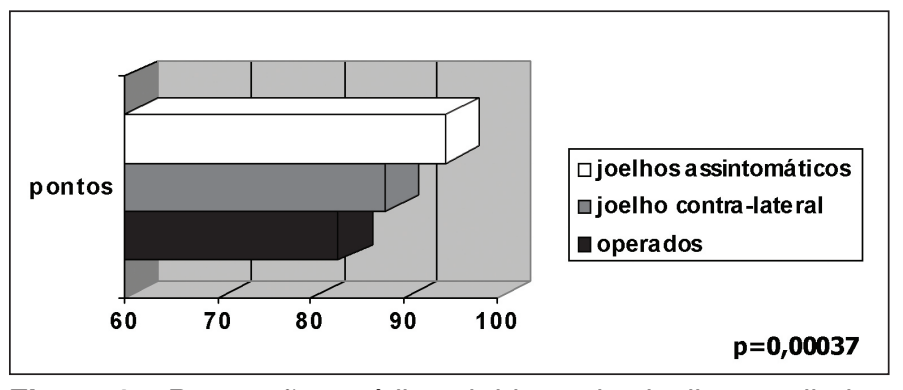

Figura 1 - Pontuações médias obtidas pelos joelhos avaliados

O objetivo deste trabalho é descrever os resultados funcionais do tratamento cirúrgico de pacientes portadores de instabilidade fêmoro-patelar habitual ou recidivante submetidos a realinhamento do aparelho extensor.

\section{MÉTODOS}

Entre fevereiro e março de 2006, foram coletados dados de pacientes pós-operados de instabilidade fêmoro-patelar. Trinta e seis pacientes portadores de instabilidade foram submetidos a tratamento cirúrgico entre dezembro de 1989 e fevereiro de 2004; 28 pacientes foram incluídos no estudo, totalizando 34 joelhos (18 esquerdos e 16 direitos), sendo seis casos operados bilateralmente. O tempo de seguimento máximo foi de 16 anos e três meses, o mínimo de dois anos, com média de seis anos e cinco meses ( $\mathrm{DP}=4,75$ anos). Vinte e dois pacientes eram do sexo feminino e seis do masculino e a idade na época da cirurgia variou entre 16 e 50 anos, com média de 27,52 anos ( $\mathrm{DP}=9,78$ anos, $\mathrm{EP}=1,67$ ano). Oito joelhos $(23,5 \%)$ apresentaram três ou menos episódios de luxação fêmoro-patelar antes do tratamento, cinco (14,5\%), entre quatro e dez episódios; e 21 $(61,8 \%)$, mais de dez episódios documentados.

Pacientes com artrose fêmoro-patelar no momento do diagnóstico, os submetidos apenas ao realinhamento proximal ou outras cirurgias prévias no mesmo joelho, foram excluídos do estudo. Não se conseguiu contato com oito pacientes na época da avaliação.

Todos os pacientes foram operados pelo mesmo cirurgião e submetidos ao realinhamento proximal e distal; 23 joelhos foram operados pela técnica de ElmslieTrillat e 11 submetidos à medialização e distalização da tuberosidade tibial anterior.

Foi aplicado um questionário desenvolvido e validado por Kujala et $a l^{(10)}$, especificamente para avaliação de patologias da articulação fêmoro-patelar, que era preenchido pelo paciente na presença de dois examinadores ou por telefone pelos que moravam em outras localidades.

Os questionários foram analisados através de programa preestabelecido (Epiinfo $98^{\circledR}$ ), sendo realizadas regressões estatísticas, teste do qui-quadrado $\left(x^{2}\right)$ e análise do valor $\mathrm{p}$ e seus valores comparados com pontuações obtidas de joelhos normais. Para critérios de comparação, as pontuações obtidas pelo grupo operado foram confrontadas com as de dois outros grupos: 22 joelhos não operados, avaliados nos pacientes operados unilateralmente, e 34 joelhos, em 17 pacientes, sem queixas ortopédicas.

\section{RESULTADOS}

O somatório geral do índice Kujala foi mensurado. A média de pontuação foi de 82,94 pontos $(p=0,00037)$. A média de pontuação nos demais grupos, respectivamente, foi de 88,81 e 94,58 (joelhos contralaterais não operados e pacientes sem sintomas, respectivamente) $(\mathrm{p}=0,00037)$ (Figura 1). As variáveis do protocolo de Kujala foram analisadas separadamente.

Após a cirurgia, 31 (91,17\%) joelhos não apresentavam claudicação, em dois $(5,8 \%)$ ela estava presente de forma leve e ocasional e em um (2,94\%), de forma constante $(p=0,93)$.

Trinta e três joelhos (97\%) apresentavam apoio total possível e sem dor; um joelho (3\%), apoio total doloroso. Nenhum paciente respondeu ser impossível o apoio total $(\mathrm{p}=0,48)$.

Em 31 joelhos $(91,17 \%)$ a capacidade de marcha era ilimitada e três $(8,83 \%)$ apresentavam alguma limitação sem, no entanto, haver impedimento de deambular menos que dois quilômetros $(\mathrm{p}=0,52)$.

Perguntados os pacientes sobre subir ou descer escadas, 26 joelhos (76,47\%) não apresentavam dificuldade ao executar tal tarefa, enquanto em seis $(17,64 \%)$ havia dor leve ao descer escadas e dois $(5,8 \%)$ tinham dor ao subir e descer. Nenhum joelho foi incapaz de realizar tal tarefa $(\mathrm{p}=0,53)$.

Com relação à capacidade de agachamento, os dados são ilustrados na figura $2(p=0,011)$. Com relação à prática de corridas, os dados são demonstrados na figura $3(p=0,012)$.

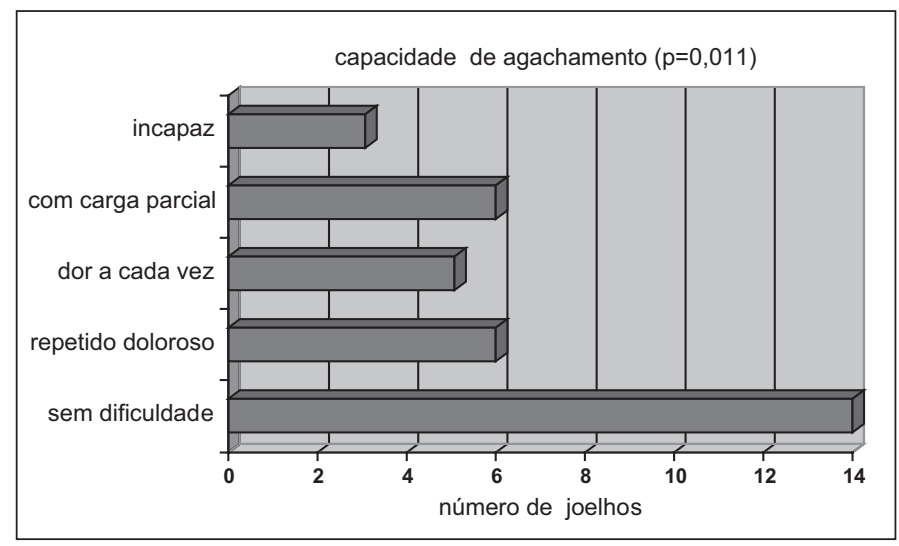

Figura 2 - Capacidade de agachamento no grupo operado 


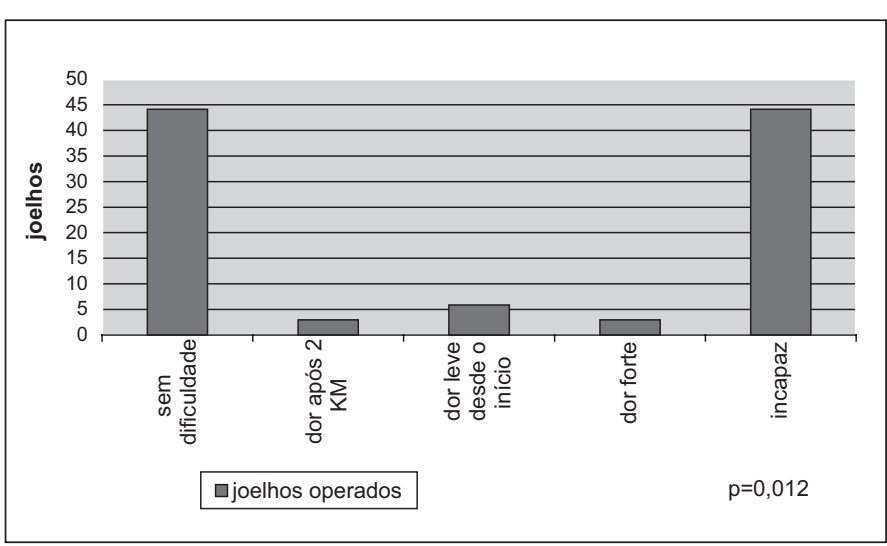

Figura 3 - Capacidade de praticar corridas no grupo - em percentagem

Vinte e três joelhos $(67,64 \%)$ realizavam salto sem dificuldade, sete $(20,58 \%)$ o faziam com pequena dificuldade e quatro $(11,76 \%)$ eram incapazes de realizar tal tarefa $(p=0,009)$.

Outro tópico abordado pelo questionário foi: dor percebida pelo paciente. Os dados são abordados na figura $4(\mathrm{p}=0,06)$.

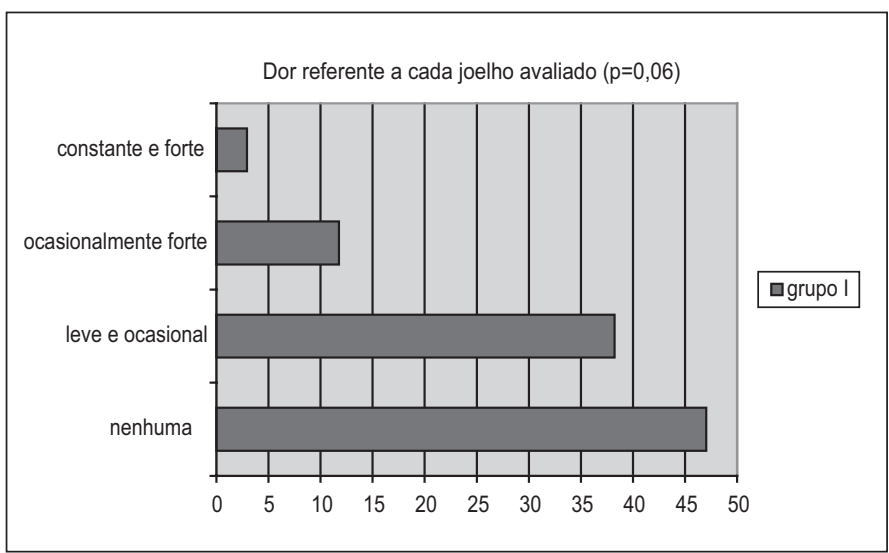

Figura 4 - Distribuição da dor no grupo avaliado - em porcentagem

Com relação ao aumento de volume do joelho, os dados são apresentados na figura $5(\mathrm{p}=0,002)$.

Ao se questionar sobre movimentos anormais da patela, incluindo luxação, os dados são demonstrados na figura $6(\mathrm{p}=0,02)$.

Quanto à limitação de flexão do joelho, 30 joelhos $(88,23 \%)$ do grupo não apresentavam déficit de flexão, em três $(8,82 \%)$ havia déficit leve (menor ou igual a cinco graus) e em um paciente $(2,94 \%)$, déficit de 15 graus $(\mathrm{p}=0,12)$.

\section{DISCUSSÃO}

A instabilidade fêmoro-patelar, embora pouco frequente, pode ser limitante, levando o paciente ao afas-

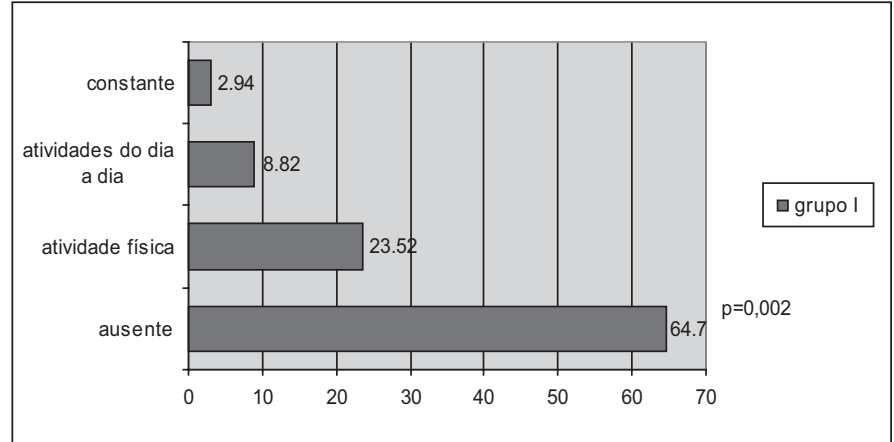

Figura 5 - Percepção de aumento do volume dos joelhos - em porcentagem

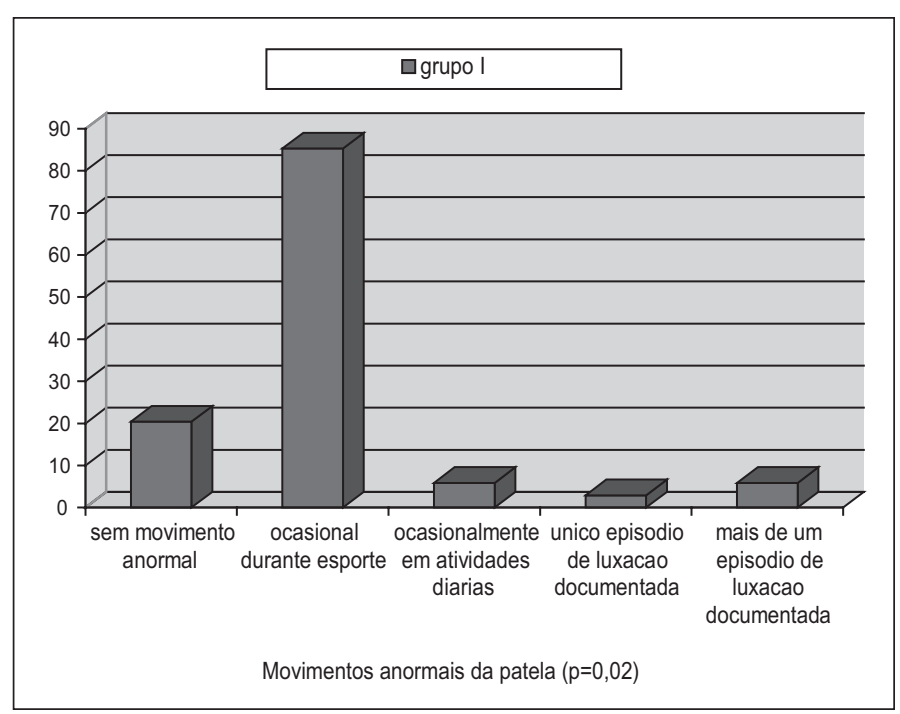

Figura 6 - Distribuição de movimentos anormais da patela entre o grupo - em porcentagem.

tamento de suas atividades de rotina, devido à apreensão de que outro episódio possa ocorrer ${ }^{(2,5,11,12)}$.

Devido a essa pouca frequência, o número de casos cirúrgicos apresentados na literatura é pequeno, o que torna relevante o conjunto deste estudo. Dantas et al $\left(^{(13)}\right.$ apresentaram 19 pacientes ( 24 joelhos) tratados com liberação retinacular e realinhamento distal (anteromedialização), Carney et $a l^{(14)}, 18$ casos tratados pela técnica de Elmsllie-Trillat, e Karataglis et a $l^{(15)}$, 49 joelhos submetidos à técnica de Elmslie-Trillat (Figura 7).

$\mathrm{Na}$ literatura, existe clara predileção pelo sexo feminino $^{(9,11)}$, o que está de acordo com os achados deste estudo. Tal predileção ocorre especialmente em jovens praticantes de esporte, sendo a diferença muscular, o condicionamento físico, a anatomia local e o aumento do ângulo Q considerados possíveis fatores causais ${ }^{(11)}$.

Pela análise das variáveis de forma isolada, as menores pontuações no grupo ocorreram nos quesitos de maior exigência articular, como agachamento, hábito de praticar corridas e prática de saltos, todos com valores 


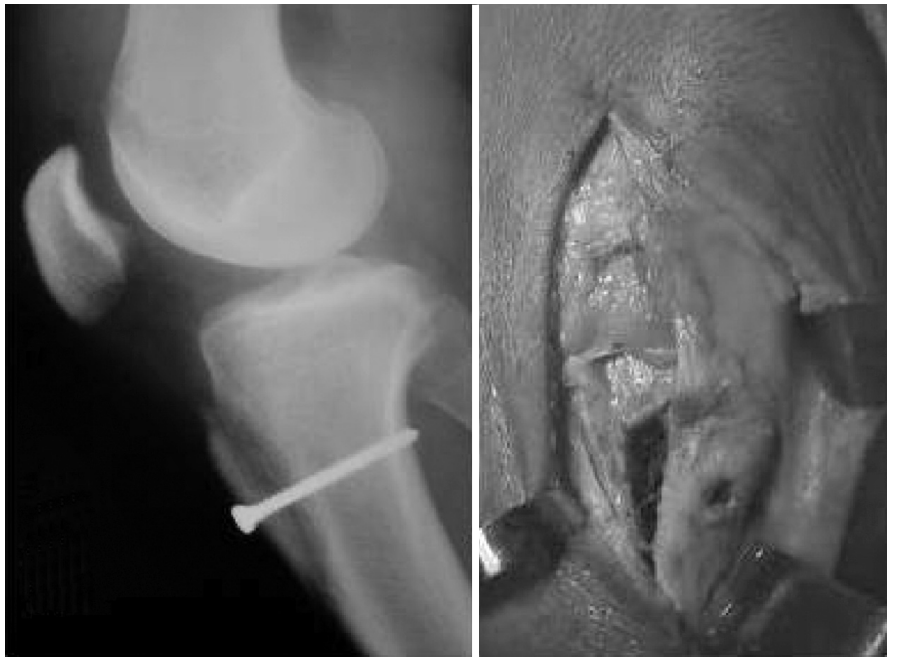

Figura 7 - Realinhamento distal pela técnica de Elmslie-Trillat A) aspecto radiológico; B) aspecto peroperatório.

estatisticamente significativos. Não foi encontrada, na literatura pesquisada, referência científica que abordasse essa observação.

A diversidade de protocolos de avaliação empregados nas pesquisas dificulta comparação de resultados na literatura. Dantas et al ${ }^{(13)}$, aplicando o questionário Lysholm, observaram melhora funcional em relação ao pré-operatório após procedimento de Elmslie-Trillat. Karataglis et $a l^{(15)}$, em 49 joelhos tratados pela mesma técnica, observaram $64,9 \%$ de resultados excelentes e bons ${ }^{(12)}$.

$\mathrm{O}$ índice de reluxação neste estudo foi de $5,88 \%$, próximo ao encontrado por Carney et $a l^{(14)}$, que foi de $7 \%$ em 18 casos. Na série de Dantas et al ${ }^{(13)}$, nenhum paciente apresentou recidiva de luxação.

A predisposição de pacientes portadores de patologia fêmoro-patelar em desenvolver artrose, com ou sem tratamento cirúrgico, é outro ponto abordado pela literatura ${ }^{(3,16-19)}$.

\section{REFERÊNCIAS}

1. Pozzi JFA, Konkewicz ER, Nora B. Tratamento cirúrgico das instabilidades rotulianas. Rev Bras Ortop. 1993;28(5):277-83.

2. Arnbjornsson A, Egung N, Rydling $O$, Stockerup $R$, Ryd L. The natural history of recurrent dislocation of the patella: long-term results of conservative and operative treatment. J Bone Joint Surg Br. 1992;(74):140-2.

3. Arendt E. Anatomy and malalignment of the patellofemoral joint: its relation to patellofemoral arthrosis. Clin Orthop Relat Res. 2005;(436):71-5.

4. Pagnano MW, Lee GC, Kelly MA. Surgical techniques for chronic lateral patellar instability. Techn Knee Surg. 2004;3(2):77-88.

5. Balsini N, Balsini NE. Instabilidade lateral da patela: tratamento cirúrgico combinado proximal via artroscópica e distal via aberta. Rev Bras Ortop. 1996;31(4):303-8.

6. Dupont JY. Pathologie douloureuse fémoro-patellaire; analyse et classification. J Traumatol Sport. 1997;14(1):30-48

7. Dejour H, Walch G, Nove-Josserand L, Guier C. Factors of patellar instability: an anatomic radiographic study. Knee Surg Sports Traumatol Arthrosc. 1994;2(1):19-26.

8. Fithian DC, Paxton EW, Cohen AB. Indications in the treatment of patellar instability. J Knee Surg. 2004;17:(1):47-56.

9. Erasmus PJ. Treatment of patellar instabilities. J Bone Joint Surg Br. 2004;86 (Suppl 4):452.

10. Kujala UM, Jaakkola LH, Koskinen SK, Taimela S, Hurme M, Nelimarkka O. Scoring of patellofemoral disorders. Arthroscopy. 1993;9(2):159-63.

11. Grelsamer RP. Patellar malalignment. J Bone Joint Surg Am. 2000;82(11):1639-50.
Alguns autores relatam artrose em até $80 \%$ dos casos, devido ao aumento da tensão no ligamento patelar após osteotomias da tuberosidade anterior da tíbia ${ }^{(3,12,14)}$. Dos pacientes operados, nenhum desenvolveu artrose fêmoro-patelar até o momento do presente estudo, o que vai contra os achados de Arnbjornsson et $a l^{(2)}$, que evidenciaram alterações degenerativas em $42 \%$ dos joelhos operados, incidência maior do que aquela no joelho contralateral com instabilidade, porém, não submetido à correção cirúrgica.

Os bons resultados funcionais após breve período de seguimento podem deteriorar-se após acompanhamento prolongado, conforme os estudos de Nakagawa et al ${ }^{(16)} \mathrm{e}$ de Carney et al ${ }^{(14)}$, que relataram 18 e 26 anos de acompanhamento, respectivamente. Como a média de seguimento deste estudo é de seis anos e cinco meses, espera-se deterioração dos resultados com o tempo, o que estimula uma nova avaliação no futuro. Gomes et al ${ }^{(20)}$, em sua série de casos tratados pela técnica de Elmslie-Trillat (20 pacientes) acompanhados por 10 anos, não observaram deterioração dos resultados durante o período de avaliação, com ótimos e bons resultados em $85 \%$ dos pacientes acompanhados.

\section{CONCLUSÃO}

Os joelhos operados apresentaram menor capacidade de executar atividades com maior demanda funcional, quando comparados com os não operados contralaterais e com os de pacientes sem queixas relacionadas com o joelho.

O objetivo principal do realinhamento do mecanismo extensor, que é a estabilização da patela, foi atingido no grupo operado, demonstrado pelo baixo índice de reluxação.

Maior período de seguimento de estudos prospectivos são necessários para avaliação do impacto do procedimento de realinhamento na prevenção ou predisposição à degeneração articular.
12. Senavongse W, Amis AA. The effects of articular, retinacular, or muscular deficiencies on patellofemoral joint stability. J Bone Joint Surg Br. 2005;87(4):577-82.

13. Dantas $P$, Nunes $C$, Moreira J, Amaral LB. Antero-medialisation of the tibial tubercle for patellar instability. Int Orthop. 2005;29(6):390-1.

14. Carney JR, Mologne TS, Muldoon M, Cox JS. Long-term evaluation of the RouxElmslie-Trillat procedure for patellar instability: a 26 -year follow-up. Am J Sports Med. 2005;33(8):1220-3.

15. Karataglis D, Green MA, Learmonth DJA. Functional outcome following modified Elmslie-Trillat procedure. Knee. 2006;13(6):464-8.

16. Nakagawa K, Wada $Y$, Minamide M, Tsuchiya A, Moriya H. Deterioration of longterm clinical results after the Elmslie-Trillat procedure for dislocation of the patella. J Bone Joint Surg Br. 2002;84:(6):861-4

17. Utting MR, Davies G, Newman JH. Is anterior knee pain a predisposing to patellofemoral osteoarthritis? Knee. 2005;12(5):362-5

18. Marcacci M, Zaffagnini S, lacono F, Visani A, Petitto A, Neri NP. Results in the treatment of recurrent dislocation of the patella after 30 years' follow-up. Knee Surg Sports Traumatol Arthrosc. 1995;3:(3):163-6.

19. Jackson AM. Anterior knee pain. J Bone Joint Surg Br. 2001;83(7):937-48

20. Gomes JLE, Sanhudo JAV, Marczyk LRS, Guerra M, Essaca PMA. Avaliação a longo prazo da instabilidade femoropatelar tratada pela técnica de Elmslie-Trillat. Rev Bras Ortop. 1996;31(7):595-9. 